\title{
Influenza and pneumococcal vaccination in hospitalized adults with pandemic (H1N1) 2009: Immunization practices and impact on clinical presentation and outcomes
}

\author{
Payeras $\mathrm{A}^{1,}{ }^{*}$, Riera $\mathrm{M}^{2}$, Viasus $\mathrm{D}^{3}$, Juan Gálvez-Acebal $\mathrm{J}^{4}$, Oteo $\mathrm{JA}^{5}$, Gracia-Ahufinger $\mathrm{I}^{6}$, Caro-Bragado $\mathrm{S}^{7}$, Cordero $\mathrm{E}^{8}$, \\ Martínez-Montauti $\mathrm{J}^{9}$, Fariñas $\mathrm{MC}^{10}$ and Carratalà ${ }^{3}$ \\ List of author affiliations are available at the end of the article \\ The Influenza A(H1N1) Study Group of the Spanish Network for Research in Infectious Diseases (REIPI) [Appendix]
}

\begin{abstract}
The purpose of this study was to determine vaccination rates against influenza and pneumococcus in a large cohort of adults hospitalized with confirmed influenza A(H1N1)pdm09 infection, and to identify differences in clinical features, frequency of bacterial coinfection, and outcomes between vaccinated and non-vaccinated patients. A prospective cohort study conducted at 14 teaching hospitals in Spain. Adult patients $(n=699)$ hospitalized with confirmed influenza A(H1N1)pdm09 infection during the pandemic and the first post pandemic influenza periods were included. Vaccination status against influenza and pneumococcus was established in 547 and 587 individuals, respectively. The vaccination rates were low: $14 \%$ for seasonal influenza and 5.4\% for pneumococcus. Despite conducting whole population and subgroup multivariate analyses, we observed no beneficial effects on outcomes (intensive care unit admission and mortality) for both vaccines. However, patients who had been vaccinated, whether against influenza or pneumococcus, did tend to develop fewer bacterial coinfections, particularly that due to $S$. pneumoniae.
\end{abstract}

Keywords: influenza A (H1N1); seasonal influenza; vaccination; pneumococcal vaccine; outcome; mortality

\section{Introduction}

In seasonal flu, as during previous influenza pandemics, an important number of clinical complications are not directly related to viral infection but, rather, to a bacterial superinfection. Streptococcus pneumoniae has been documented as the main agent responsible for secondary bacterial pneumonia [1, 2]. During the 1918 influenza pandemic most of the deaths were due to bacterial pneumonia, with bacteremia caused by $S$. pneumoniae being documented in nearly half of them [3]. Although the prevalence of bacterial coinfection was relatively low during the recent pandemic (H1N1) 2009, it was nonetheless an independent factor for severe disease [4]. Moreover, S. pneumoniae was the most frequently isolated microorganism in bacterial pneumonia complications during the pandemic period, as well as in the first post-pandemic influenza season [4-6]. The synergistic interaction between bacteria and the influenza virus has been shown to contribute to the severity of the resulting infection in animal models [7], as well as in the case of pandemic influenza $A(H 1 N 1)$ pdm09 infection [8].

Seasonal influenza and 23-valent polysaccharide pneumococcal vaccines are recommended for older patients and those with comorbid conditions such as lung, heart, hepatic, or kidney diseases and immunodeficiency states [9]. The immunogenicity, safety, and efficacy of influenza $A(H 1 N 1) p d m 09$ vaccines have been described in different populations [10-14]. However, vaccination rates are far from optimum $[15,16]$, with possible factors influencing vaccination being related both to physicians and to the psychosocial environment of the patients $[17$,

*Corresponding author: Dr. Antoni Payeras, Department of Internal
Medicine, Hospital Son Llatzer, Carretera Palma-Manacor Km 4, 07198,
Palma de Mallorca, Illes Balears, Spain, Tel.: +34-871202035; Fax: +34-
871202352. E-mail: apayeras@hsll.es Received 27 August 2013 Revised 3 November 2013 Accepted 10 November 2013 Published 17 November 2013

Citation: Payeras A, Riera M, Viasus D, Juan Gálvez-Acebal J, Oteo JA, Gracia-Ahufinger I, Caro-Bragado S, Cordero E, Martínez-Montauti J, Fariñas MC, Carratalà J (2013) Influenza and pneumococcal vaccination in hospitalized adults with pandemic (H1N1) 2009: Immunization practices and impact on clinical presentation and outcomes. J Vaccines Immun 1:22-31. doi:10.14312/2053-1273.2013-4

Copyright: (C) 2013 Payeras A, et al. This is an open-access article distributed under the terms of the Creative Commons Attribution License, which permits unrestricted use, distribution and reproduction in any medium, provided the original author and source are credited 
18]. The previous trivalent 2008-2009 seasonal influenza vaccination has demonstrated moderate protection against laboratory-confirmed pandemic influenza A (H1N1) 2009 associated illness [19]. In addition, during the pandemic, dual vaccination with both H1N1 and seasonal vaccines reduced mortality and hospitalization rates in the institutionalized elderly [20], and dual vaccination of influenza and pneumococcus reduced mortality among nursing home older adults infected with the pandemic influenza A (H1N1) 2009 virus [21]. However, the available information remains controversial and scarce, and more studies are therefore needed to corroborate these findings in other settings and with different populations.

The aims of the present study were to determine vaccination rates against influenza or $S$. pneumoniae in a large cohort of hospitalized adults with confirmed influenza A(H1N1)pdm09 infection, and to identify differences in clinical features, frequency of bacterial coinfection, and outcomes between vaccinated and nonvaccinated patients.

\section{Methods}

\section{Setting, patients, and study design}

This is an observational prospective cohort study conducted at 14 teaching hospitals belonging to the Spanish Network for Research in Infectious Diseases (REIPI). All adult patients ( $>18$ years old) hospitalized with confirmed influenza A(H1N1)pdm09 infection during the pandemic period (April to November 2009) and those hospitalized with pneumonia during the first post-pandemic influenza period (December 2010 to March 2011) were prospectively recruited and followed up.

A confirmed case was defined as a person with an influenza-like illness with confirmed laboratory influenza A(H1N1)pdm09 virus infection documented by real-time polymerase chain reaction (RT-PCR) or viral culture. Influenza A(H1N1)pdm09 virus testing was performed in each institution.

The study was approved by the Ethics Committee of the coordinating center, the Hospital Universitari de Bellvitge, and written informed consent was obtained from patients.

\section{Clinical assessment and follow-up}

Patients were seen during their hospital stay by one or more of the researchers in each participating hospital, who recorded clinical data in a standardized, computerassisted protocol. Data were collected on demographic characteristics, pneumococcal and influenza vaccination, comorbidities, clinical signs and symptoms, biochemical analysis, chest X-ray findings, concomitant and/or secondary bacterial pneumonia or infection, antiviral and antibiotic treatment, time to clinical stability, and outcomes, including mortality during hospitalization.

Completed protocols were sent to the coordinating center and all the data were revised by two senior investigators prior to the final validation.

\section{Definitions}

Patients were considered to be pneumococcal-vaccinated if 23-valent polysaccharide pneumococcal vaccine had been administered in the five years before admission, and influenza-vaccinated if seasonal influenza vaccine had been administered during the year prior to admission. Vaccination status was determined through interviews with patients or their relatives, as well as from reviews of hospital and personal health records (vaccination card). Pneumonia was defined as the presence of a new infiltrate on a chest radiograph.

Bacterial coinfection was considered to be present in patients who showed positive bacterial blood or respiratory cultures or positive urinary antigen tests for $S$. pneumoniae or Legionella pneumophila serogroup 1. The study of pathogens in sputum, pleural effusion, bronchoalveolar lavage, and blood was performed by standard microbiologic procedures. Standard serologic methods were used to determine antibodies against atypical agents.

The Pneumonia Severity Index (PSI) [22] and CURB65 [23] were calculated in each pneumonia episode. Complications were defined as any untoward circumstances occurring during hospitalization. Time to clinical stability was evaluated as described elsewhere [24]. Overall mortality was defined as death from any cause during hospitalization.

The hospital admission criteria, microbiologic studies, intensive care unit (ICU) admission criteria, and treatment decisions were not standardized and were the responsibility of the attending physician.

\section{Statistical analysis}

The results were analyzed using a commercially available statistical software package (SPSS, version 15.0; SPSS Inc., Chicago, Illinois). Descriptive statistics were obtained for all study variables, with all proportions being calculated as percentages of the patients with available data.

We compared the clinical characteristics and outcomes between patients who were vaccinated and those who were not vaccinated against influenza and/or $S$. pneumoniae. The same analysis was performed in patients with vaccine indication according to guidelines, and in those with pneumonia. Significant differences between groups were assessed by means of the chi-square test or Fisher's exact test for categorical variables, as appropriate. Either the Student's t test or the Mann-Whitney test was applied for continuous variables. For outcome variables 
we also calculated the corresponding odds ratio with 95\% confidence interval.

Logistic regression multivariate models were developed for each of the following outcomes: bacterial coinfection, ICU admission, and overall mortality. These multivariate analyses were performed for the whole population and for patients with vaccine indications according to established guidelines. Variables included in the models were influenza or pneumococcal vaccination and all those variables shown to be statistically related with the corresponding outcome variable in the bivariate analysis.

Statistical significance was set at $\alpha=0.05$. All reported $P$ values are 2 -tailed.

\section{Results}

General characteristics of the study population and vaccination rates

A total of 699 adults with influenza A(H1N1)pdm09 infection required hospitalizationduring thestudy periods, 585 in the pandemic period and 114 in the first postpandemic period. Of these, $350(50.1 \%$ ) had a respiratory tract infection without radiological consolidation and 349 (49.9\%) were diagnosed with pneumonia. Demographic characteristics and comorbid conditions for the whole population are summarized in Table 1.

The vaccination rate was $14 \%$ for influenza (77 of 547 with available information on vaccination status) and $5.4 \%$ for pneumococcus (32 of 587 with available information). There were no demographic or clinical differences between patients whose vaccination status was known and those in whom it was not established. According to guidelines, 464 (66.4\%) of the 699 patients met at least one criterion for administration of influenza and/or pneumococcal vaccines.

Sixty (8.5\%) patients had a microbiologically documented bacterial co-infection (Table 2). S. pneumoniae was documented as the causative pathogen in 41 (5.8\%) patients (6 cases had positive sputum culture, 5 bacteremia, and 31 positive urinary antigen test).

At least one complication developed in 137 (12.5\%) patients, and it was mainly of a respiratory nature (71 needed invasive ventilation, 50 noninvasive ventilation, 53 showed respiratory distress, 35 pleural effusion, and 3 empyema). Thirty-five (5\%) cases presented with septic shock at admission, 119 (17\%) patients required ICU admission, and 38 (5.4\%) died during hospitalization.

Comparison of clinical and prognostic features between patients who were vaccinated versus not vaccinated against influenza

Among 547 patients with a known influenza vaccination status, 77 had received influenza vaccine. Immunized patients were older, more often male, and were more
Table 1 Demographic and clinical characteristics for the whole study population.

\begin{tabular}{|c|c|}
\hline Variable & Total $n=699(\%)$ \\
\hline Male/female & $367(52.5) / 332(47.5)$ \\
\hline Age in years (median) & 41 (range: 16-97) \\
\hline Smoker (active) & $221(31.6)$ \\
\hline Alcohol use (active) & $46(6.6)$ \\
\hline Comorbidity & $386(55.2)$ \\
\hline COPD & $69(9.9)$ \\
\hline Asthma & $108(15.5)$ \\
\hline Diabetes & 75 (10.7) \\
\hline Diabetes (complicated) & $25(3.6)$ \\
\hline Heart disease & $54(7.7)$ \\
\hline Heart failure & $26(3.7)$ \\
\hline Coronary artery disease & $17(2.4)$ \\
\hline Cerebrovascular disease & $14(2)$ \\
\hline Hemiplegia & $4(0.6)$ \\
\hline Dementia & $17(2.4)$ \\
\hline Peripheral vascular disease & $8(1.1)$ \\
\hline \multicolumn{2}{|l|}{ Chronic kidney disease: } \\
\hline Mild-moderate & $20(2.9)$ \\
\hline Severe & $17(2.4)$ \\
\hline Peptic gastric ulcer & $9(1.3)$ \\
\hline \multicolumn{2}{|l|}{ Hepatopathy: } \\
\hline Mild-moderate & $27(3.9)$ \\
\hline Severe & $11(1.6)$ \\
\hline HIV/AIDS & $31(4.4)$ \\
\hline Connective tissue disease & $16(2.3)$ \\
\hline Solid tumor (localized) & $21(3)$ \\
\hline Solid tumor (metastasis) & $19(2.7)$ \\
\hline Hematological malignancy & $18(2.6)$ \\
\hline Solid organ transplant & $22(3.1)$ \\
\hline Bone marrow transplant & $6(0.9)$ \\
\hline Any other immunodeficiency* & $52(7.4)$ \\
\hline Pregnant & $100(14.3)$ \\
\hline Nursing home resident & $11(1.6)$ \\
\hline
\end{tabular}

*Includes: neutropenia, chemotherapy, corticosteroids, or other immunosuppressive drugs. 
Table 2 Etiology of bacterial co-infections for the whole population

\begin{tabular}{ll}
\hline Bacterial isolate & Total $n=699(\%)$ \\
\hline S. pneumoniae & $37(5.3)$ \\
S. pneumoniae and other* & $4(0.6)$ \\
H. influenzae & $4(0.6)$ \\
P. aeruginosa & $4(0.6)$ \\
S. aureus & $2(0.3)$ \\
M. catarrhalis & $2(0.3)$ \\
A. baumanii & $2(0.3)$ \\
Others** & $6(0.8)$ \\
\hline
\end{tabular}

${ }^{*}$ S. aureus (2) and S. mitis (1), H. parainfluenzae (1); ** S. maltophila (1), S. pyogenes (2), S. sanguis (2); Positive blood cultures: $S$ pneumoniae (5), S. sanguis (2), S. pyogenes (1), S. aureus (1), A. baumanii (1), $H$. parainfluenzae (1), unknown (1). likely to have chronic comorbid conditions (Table 3). Figure 1 displays the main differences in comorbidity rates between vaccinated and non-vaccinated patients. The main clinical features, complications, and prognostic variables for these groups are summarized in Table 3.

Three (3.8\%) influenza-immunized and 37 (7.8\%) non-influenza immunized patients developed bacterial coinfection, of which, respectively, 2 (2.5\%) and 23 (4.8\%) were caused by $S$. pneumoniae. None of the vaccinated patients developed bacteremia or empyema. However, in the unvaccinated group, 7 developed bacteremia (2 by $S$. pneumoniae) and 1 empyema by $S$. pyogenes. These differences did not reach statistical significance. Use of antibiotics was more frequent in vaccinated patients [63 (81.8\%) vs. 324 (68.9\%), OR 1.98 (95\% CI 1.073.66), $\mathrm{p}=0.02]$, although not statistical differences were observed in the pneumonia frequency.

Table 3 Main demographic, clinical, and outcome differences between vaccinated and non-vaccinated patients whose vaccine status against influenza was known (whole population).

\begin{tabular}{|c|c|c|c|}
\hline Variable (Whole population) & $\begin{array}{l}\text { Vaccinated (Influenza) } \\
n=77(\%)\end{array}$ & $\begin{array}{l}\text { Not vaccinated (Influenza) } \\
n=470(\%)\end{array}$ & $\begin{array}{l}\text { Statistical significance } \\
\text { OR }(95 \% \text { CI) }\end{array}$ \\
\hline Male sex & $48(62.3)$ & $229(48.7)$ & $1.74(1.06-2.85) \mathrm{p}=0.02$ \\
\hline Age (mean) & 51.7 (SD 15.9) & 38.2 (SD 14.6) & $\mathrm{p}<0.001$ \\
\hline Any comorbidity & $69(89.6 \%)$ & $220(46.8 \%)$ & $0.1(0.04 .0 .21) \mathrm{p}<0.001$ \\
\hline Mean systolic blood pressure ( $\mathrm{mmHg}$ ) & 127.4 (SD 27.8) & 119.4 (SD 22.6) & $\mathrm{p}=0.006$ \\
\hline Serum creatinine $(\mathrm{mg} / \mathrm{dL})$ & $1.3(\mathrm{SD} 1.5)$ & 0.9 (SD 0.9) & $\mathrm{p}=0.002$ \\
\hline Serum bicarbonate (mmol/L) & 25.4 (SD 4.6) & $23.3(\mathrm{SD} 3.2)$ & $\mathrm{p}<0.001$ \\
\hline Lymphocyte count (cells/ $\mu \mathrm{L}$ ) & 1485,2 (SD 2994.4) & 1064.7 (SD 674.8) & $\mathrm{p}=0.008$ \\
\hline Dyspnea & $49(63.6)$ & $206(43.8)$ & $0.44(0.27-0.73) \mathrm{p}=0.001$ \\
\hline Baseline oxygen saturation (\%) & $91.2(\mathrm{SD} 6.2)$ & 93.4\% (SD 5.3) & $\mathrm{p}=0.002$ \\
\hline PaCO2 (mmHg) & 39.3 (SD 10.6) & 35.2 (SD 7.8 & $\mathrm{p}=0.003$ \\
\hline Abnormal resp. examination: & $21(27.2)$ & $219(46.5)$ & $2.39(1.4-4.08) \mathrm{p}=0.001$ \\
\hline Chest retraction & $7(9)$ & $16(3.4)$ & $0.35(0.14-0.9) \mathrm{p}=0.03$ \\
\hline Crackles & $36(46.7)$ & $158(33.6)$ & $0.59(0.36-0.96) \mathrm{p}=0.03$ \\
\hline Wheezing \& rhonchi & $35(45.4)$ & $119(25.3)$ & $0.4(0.24-0-66) \mathrm{p}<0.001$ \\
\hline Shock & $3(3.8)$ & $4(0.8)$ & $0.2(0.04-0.95) p=0.02$ \\
\hline ICU admission & $16(20.7)$ & $51(10.8)$ & $0.46(0.24-0.86) \mathrm{p}=0.01$ \\
\hline Respiratory complications & $15(19.4)$ & $39(8.2)$ & $0.37(0.19-0.71) p=0.002$ \\
\hline Noninvasive ventilation & $9(11.6)$ & $16(3.4)$ & $0.26(0.11-0.62) \mathrm{p}=0.001$ \\
\hline Heart failure & $4(5.1)$ & $5(1)$ & $0.19(0.05-0.74) \mathrm{p}=0.02$ \\
\hline Length of hospital stay (days) & $9.1(\mathrm{SD} 8.8)$ & $6.8(7.9)$ & $\mathrm{p}=0.02$ \\
\hline Death & $7(9)$ & $11(2.3)$ & $0.24(0.09-0.63) p=0.002$ \\
\hline
\end{tabular}


(A)
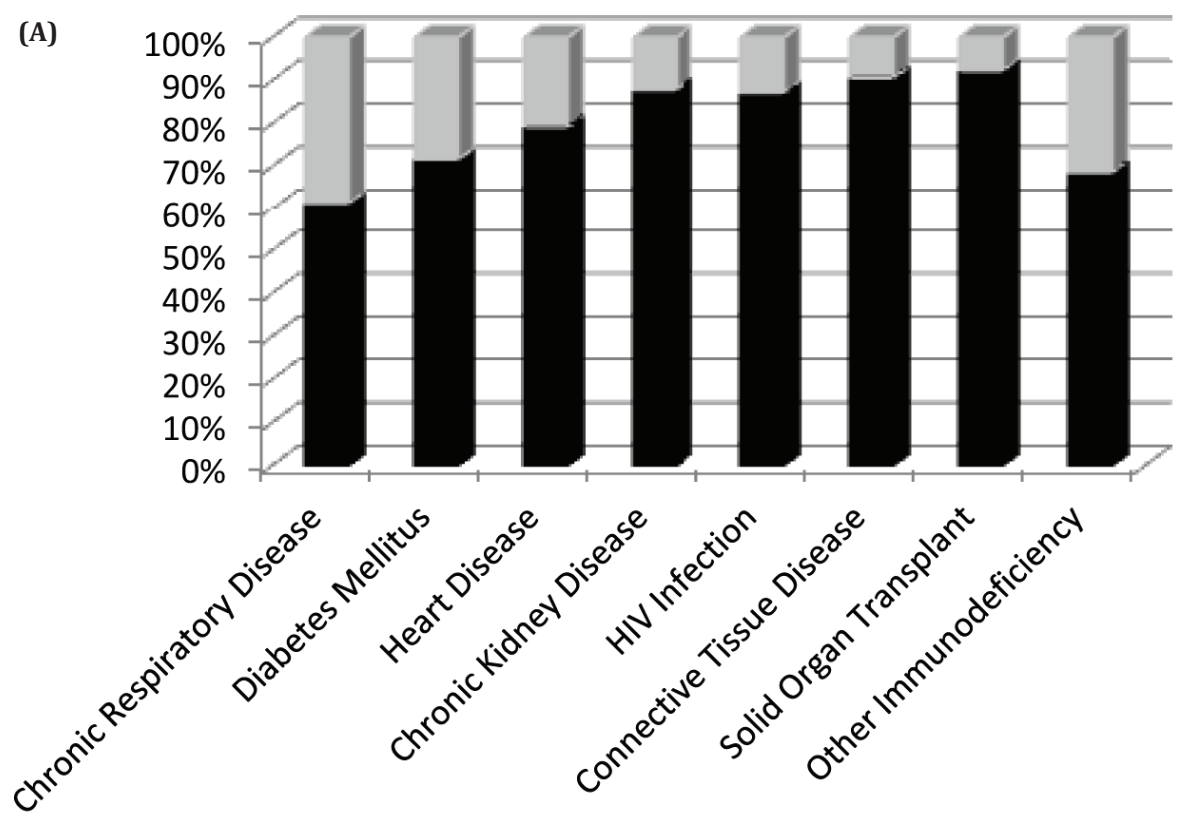

(B)

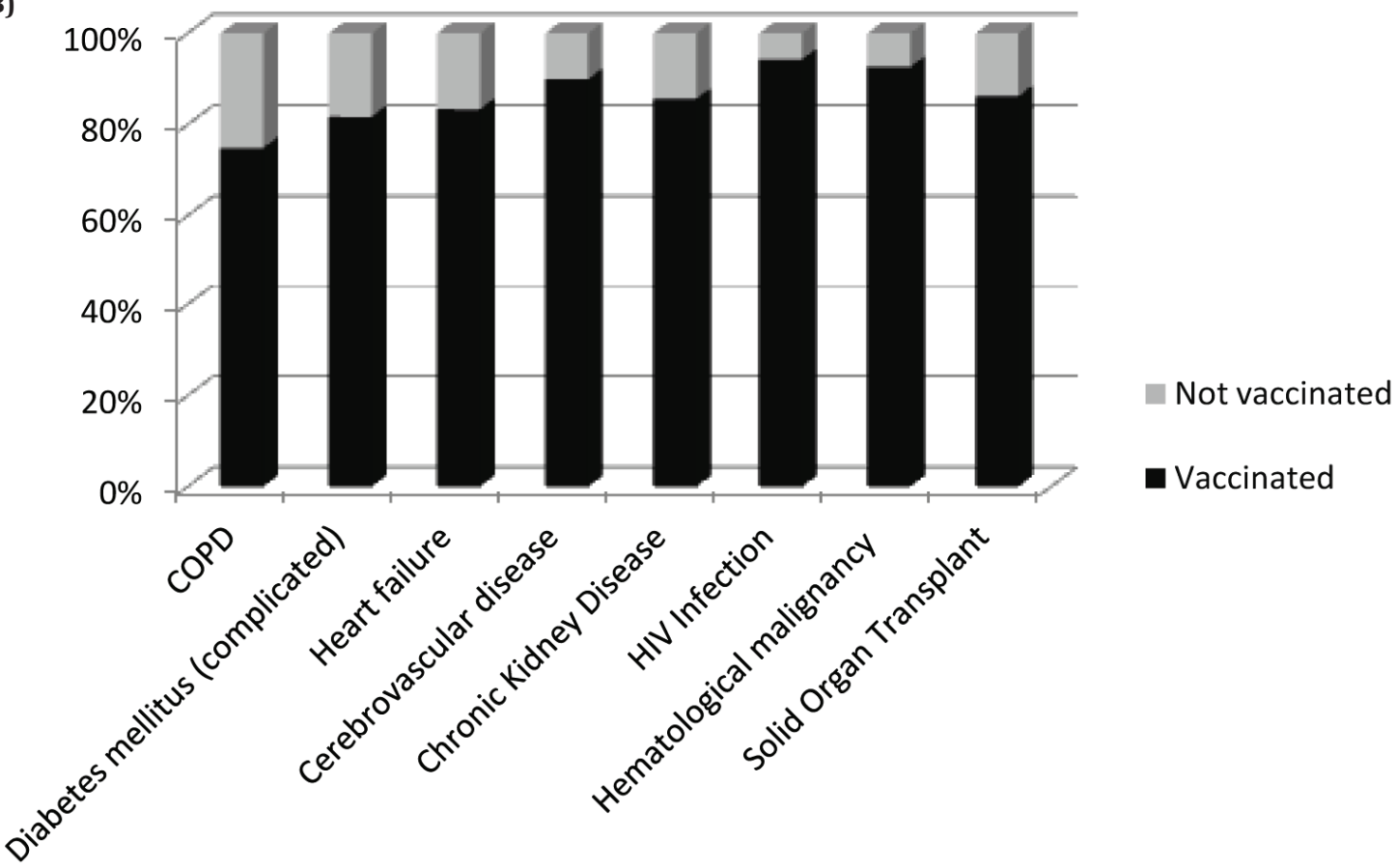

Figure 1 Influenza-vaccination (A) and pneumococcal-vaccination (B) rates according to each comorbidity (all differences are statistically significant, $\mathrm{p}<0.05)$.

When the analysis was restricted to those patients with influenza vaccine indication according to guidelines, immunized patients were older (52.3 vs. 40.6 years, $\mathrm{p}<0.001$ ) and had a longer hospital stay (mean 9.1 vs. 6.8 days, $\mathrm{p}=0.03$ ), but there were no significant differences between the two groups in terms of clinical manifestations, complications or death.

The analysis of patients with pneumonia showed that influenza-immunized patients were older and more often presented comorbidities (Table 4). Pneumonia episodes were more severe in vaccinated patients, as evidenced by PSI and CURB-65 scores. Vaccinated patients were also more likely to develop complications, mainly of a respiratory nature. A microbiologically confirmed bacterial coinfection was more common among those not immunized against influenza (2.7\% vs. $31 \%$; $p=0.03)$. The only vaccinated case with confirmed bacterial pneumonia was caused by $S$. pneumoniae, identified by a positive urinary antigen test. Among patients who were not influenza vaccinated, 21 pneumococcal pneumonias were documented (17 had a positive urinary antigen test, 5 a positive sputum culture, and 2 bacteremia). 
Table 4 Main demographic, clinical, and outcome differences between vaccinated and non-vaccinated patients whose vaccine status against influenza was known and who had a pneumonia diagnosis.

\begin{tabular}{|c|c|c|c|}
\hline Variable (patients with pneumonia) & $\begin{array}{l}\text { Vaccinated (Influenza) } \\
n=37(\%)\end{array}$ & $\begin{array}{l}\text { Not vaccinated (Influenza) } \\
n=192(\%)\end{array}$ & $\begin{array}{l}\text { Statistical significance } \\
\text { OR }(95 \% \text { CI) }\end{array}$ \\
\hline Age & $55.1(\mathrm{SD} 15.1)$ & 38.58 (SD 19.9) & $\mathrm{p}<0.001$ \\
\hline Any comorbidity & $34(91.8 \%)$ & $81(42.1 \%)$ & $0.06(0.01-0.21) \mathrm{p}<0.001$ \\
\hline \multicolumn{4}{|l|}{ Severity } \\
\hline PSI score & 87 (SD 39) & 52.7 (SD 30.2) & $\mathrm{p}<0.001$ \\
\hline CURB-65 score & 1.2 (SD 1.1) & 0.4 (SD 0.7) & $\mathrm{p}<0.001$ \\
\hline Dyspnea & $30(81)$ & $109(56.7)$ & $0.3(0.12-0.73) p=0.006$ \\
\hline Wheezing and rhonchi & $20(54)$ & $50(26)$ & $0.3(0.14-0.63) p=0.001$ \\
\hline Baseline oxygen saturation (\%) & 87.5 (SD 6.2) & $91.4(\mathrm{SD} 6.6)$ & $\mathrm{p}=0.002$ \\
\hline $\mathrm{PaCO}_{2}(\mathrm{mmHg})$ & 39.7 (SD 11) & 35.3 (SD 9.2) & $\mathrm{p}=0.03$ \\
\hline Serum bicarbonate $(\mathrm{mmol} / \mathrm{L})$ & $25.3(\mathrm{SD} 4.5)$ & $23.2(\mathrm{SD} 3.2)$ & $p=0.006$ \\
\hline Hematocrit (\%) & $37.2 \%$ (SD 5.7) & $40.4 \%$ (SD 5.2) & $\mathrm{p}=0.001$ \\
\hline Serum creatinine $(\mathrm{mg} / \mathrm{dL})$ & $1.1(\mathrm{SD} 0.6)$ & $0.9(\mathrm{SD} 0.4)$ & $\mathrm{p}=0.001$ \\
\hline Glucose (mg/dL) & 145.3 (SD 56.8) & $120.9(\mathrm{SD} 45.2)$ & $\mathrm{p}=0.005$ \\
\hline ICU admission & $14(37.8)$ & $39(20.3)$ & $0.41(0.19-0.88) \mathrm{p}=0.02$ \\
\hline Respiratory complications & $13(35.1)$ & $30(15.6)$ & $0.34(0.15-0.74) p=0.005$ \\
\hline Noninvasive ventilation & 7 (18.9) & $14(7.2)$ & $0.33(0.12 .0 .9) \mathrm{p}=0.02$ \\
\hline Death & $7(18.9)$ & $11(5.7)$ & $0.26(0.09-0.72) p=0.01$ \\
\hline
\end{tabular}

Comparison of clinical and prognostic features between patients who were vaccinated versus not vaccinated against S. pneumoniae.

Among 587 patients with a known pneumococcal vaccine status, 32 had been vaccinated. The majority of them were men and they were older and more likely to have chronic comorbid conditions (Figure 1). They also had a shorter time between symptom onset and hospital admission (2.7 vs. 3.7 days, $p=0.04$ ), as well as higher lymphocyte counts, $\mathrm{PaCO} 2$, and serum bicarbonate. No other differences in clinical manifestations or laboratory values were observed (Table 5). One (3.1\%) vaccinated patient had a microbiologically confirmed bacterial coinfection (sputum culture positive for Pseudomonas aeruginosa), compared with $45(8.1 \%)$ bacterial coinfections in non-vaccinated patients $(68.8 \%$ were due to $S$. pneumoniae). Although vaccinated patients more often required noninvasive ventilation, no other differences were observed in terms of complications or mortality rates between the two groups (Table 5).

In the sub-analysis of the 294 patients with pneumonia, $16(5.4 \%)$ had received pneumococcal vaccine, all of them with vaccine indication according to guidelines. As previously observed those immunized were mainly male, older, and more likely to present chronic comorbid conditions. They also had lower baseline oxygen saturation and higher levels of $\mathrm{PaCO}_{2}$ and serum bicarbonate. Pneumonia episodes were more severe in vaccinated patients, as evidenced by PSI (93.6 vs. 62.5 points, $\mathrm{p}=0.001$ ) and CURB-65 (1.4 vs. 0.6 points, $\mathrm{p}<0.001)$ scores. Finally, noninvasive ventilation was more frequently required by these patients, but no differences were observed for the other outcome variables (Table 5). Immunized subjects with pneumonia did not have any microbiologically documented pneumococcal infection or bacteremia, compared, respectively, with 28 (10\%) and 4 (1.4\%) of non-immunized patients ( $p>0.05$ in each case).

Clinical and prognostic differences between patients vaccinated versus not vaccinated with both vaccines (influenza and S. pneumoniae)

All of the 28 patients who received both vaccines had vaccine indications according to guidelines. As in the previous analysis, vaccinated subjects were mainly men and were older than non-vaccinated patients, and they were also more likely to have chronic comorbid conditions. They also presented lower baseline oxygen saturation and an increased need for noninvasive ventilation. In cases with a pneumonia diagnosis, PSI and CURB-65 scores were also higher for immunized patients (data not shown). 
Table 5 Main differences in demographic, clinical, and outcome characteristics among patients with known vaccination status against Streptococcus pneumoniae, for the whole population and for those with a pneumonia diagnosis.

\begin{tabular}{|c|c|c|c|c|c|c|}
\hline Variable & $\begin{array}{l}\text { Vaccinated } \\
\text { Whole } \\
\text { population } n= \\
32(\%)\end{array}$ & $\begin{array}{l}\text { Not vaccinated } \\
\text { Whole } \\
\text { population } n= \\
555(\%)\end{array}$ & $\begin{array}{l}\text { Statistical } \\
\text { significance OR } \\
(95 \% \text { CI) }\end{array}$ & $\begin{array}{l}\text { Vaccinated } \\
\text { Pneumonia } n= \\
16(\%)\end{array}$ & $\begin{array}{l}\text { Not Vaccinated } \\
\text { Pneumonia } n= \\
278(\%)\end{array}$ & $\begin{array}{l}\text { Statistical } \\
\text { significance OR } \\
(95 \% \text { CI) }\end{array}$ \\
\hline Male sex & $22(68.7)$ & $279(50.2)$ & $\begin{array}{l}0.45(0.21-0.98) \\
p=0.04\end{array}$ & $14(87.5)$ & $152(54.6)$ & $\begin{array}{l}0.62(0.5-0.7) \\
p=0.01\end{array}$ \\
\hline Age (years) & 52.6 (SD 17.9) & 40.8 (SD 15.3) & $\mathrm{p}<0.001$ & 58.8 (SD 15.5) & 42.8 (SD 14.4) & $\mathrm{p}<0.001$ \\
\hline Any comorbidity & 30 (93.7) & $274(49.3)$ & $\begin{array}{l}15.3(3.64- \\
64.99) \mathrm{p}<0.001\end{array}$ & $16(100)$ & $129(46.4)$ & $\begin{array}{l}0.46(0.4-0.52) \\
\mathrm{p}<0.001\end{array}$ \\
\hline Lymphocyte count (cells/ $\mu \mathrm{L}$ ) & $\begin{array}{l}2017.9(\mathrm{SD} \\
4368.6)\end{array}$ & $\begin{array}{l}1.093 .2(\mathrm{SD} \\
1298.5)\end{array}$ & $\mathrm{p}=0.002$ & 1354.3 (SD 945) & $\begin{array}{l}1042.5(\mathrm{SD} \\
1615.3)\end{array}$ & NS \\
\hline Baseline oxygen saturation (\%) & 91.9 (SD 7) & 92.5 (SD 5.9) & NS & $86.2(\mathrm{SD} 6.6)$ & 90.5 (SD 6.8) & $\mathrm{p}=0.03$ \\
\hline $\mathrm{PaCO}_{2}(\mathrm{mmHg})$ & 41.8 (SD 12.2) & 36 (SD 8.9) & $\mathrm{p}=0.01$ & 45.4 (SD 13.1) & $36.2(\mathrm{SD} 9.8)$ & $\mathrm{p}=0.005$ \\
\hline Serum bicarbonate $(\mathrm{mmol} / \mathrm{L})$ & 25.6 (SD 5) & 23.5 (SD 3.6) & $\mathrm{p}=0.03$ & 27.2 (SD 5.4) & $23.5(\mathrm{SD} 3.6)$ & $\mathrm{p}=0.003$ \\
\hline Noninvasive ventilation & $5(15.6 \%)$ & $33(5.9 \%)$ & $\begin{array}{l}2.92(1.05-8.09) \\
p=0.04\end{array}$ & $5(31.2)$ & $30(10.7)$ & $\begin{array}{l}3.75(1.22- \\
11.55) \mathrm{p}=0.03\end{array}$ \\
\hline
\end{tabular}

None of the patients who received both vaccines had a pneumococcal infection or bacteremia, compared with 6 ( 2 with bacteremia) of the 238 (2.5\%) who were not vaccinated.

\section{Multivariate logistic regression analysis}

In the multivariate regression models, bacterial coinfection was mainly related to COPD [OR 4.15 (95\% CI 1.62-10.64), $\mathrm{p}=0.003$ ] and immunodeficiency [OR 5.6 (95\% CI 2.14-14.64), p<0.001]. ICU admission was associated with the presence of heart failure [OR 5.53 (95\% CI 1.71-17.84), $\mathrm{p}=0.004$ ] and alcohol intake [OR 3.14 (95\% CI 1.24-7.94), p=0.01). Finally, overall mortality was related with comorbidity [OR 9.12 (95\% CI 1.15-72.3), $\mathrm{p}=0.03$ ] and alcohol intake [OR 7.23 (95\% CI 1.97-26.49), $\mathrm{p}=0.003$ ). Neither the remaining variables analyzed (age, diabetes mellitus, chronic kidney disease, or being an active smoker) nor influenza ( $p>0.2)$ or pneumococcal vaccination $(p>0.6)$ were related with any of these three outcome variables.

\section{Discussion}

In this large cohort study of hospitalized patients with confirmed pandemic influenza A(H1N1)pdm09 infection, we observed low vaccination rates for both seasonal influenza (14\%) and S. pneumoniae (5.4\%), even in patients who had vaccine indications according to guidelines. In addition, despite conducting whole population and subgroup multivariate analyses, we found that seasonal influenza and pneumococcal vaccines had no beneficial effect in terms of reducing ICU admission or overall mortality. However, patients who had received vaccine (influenza and/or pneumococcal) tended to have fewer bacterial co-infections.

A significant gap between vaccination awareness and actual vaccination rates has been described for both vaccines in different settings $[15,16,25]$. In our study, patients immunized against influenza were mainly men and older; these data are not surprising since a gender effect for vaccine reception has been previously reported [26], and compliance with influenza vaccination among those under 65 years with comorbidities has varied over time, with low coverage rates of approximately 30\% [15, 27]. In our patients the chronic conditions most often related with seasonal influenza vaccine were COPD, heart disease, complicated diabetes mellitus, chronic kidney disease, HIV infection, connective tissue disease, and solid organ transplant.

Especially noteworthy is the low vaccination rate observed among pregnant women in our cohort, of whom only $2.7 \%$ had been immunized. Although pregnancy was identified as a risk factor for complications during the pandemic influenzaA (H1N1) 2009, the low rate of seasonal influenza vaccination during the 2008-2009 vaccine campaign, before the first cases of pandemic influenza (H1N1) 2009 were diagnosed in the spring of 2009, is substantially below target levels [28]. The subjective perceived low risk of infection and concerns about vaccine safety could be responsible for poorer vaccination rates in the first postpandemic season, both in pregnant women and in other risk groups. An epidemiological study of vaccination against 2009 season influenza in Spain concluded that the coverage finally achieved among people with an indication due to a chronic health condition in the H1N1 campaign was much lower than expected [29].

Another noteworthy finding is the fact that immunized patients presented more complications, mainly shock, ICU admissions, need for noninvasive ventilation, and mortality. This is probably due to a selection bias, since the baseline risk of this group was increased by their being older and having more chronic conditions. Although the efficacy and effectiveness of influenza vaccination have 
been clearly demonstrated [30, 31], a Cochrane review concluded that while influenza vaccination in patients with COPD significantly reduced acute exacerbations with an effectiveness over $60 \%$, there was no evidence of an effect on hospitalization or mortality rates [32]. In our study, neither influenza nor pneumococcal vaccination was related in the multivariate analyses with a lower rate of ICU admission, mortality, or bacterial co-infection.

As already mentioned, previous seasonal influenza vaccination, when the circulating influenza strain was not matched by the trivalent seasonal influenza vaccine, was related to better prognosis in patients infected with influenza A(H1N1) pdm09 [21]. Moreover, prior vaccination with seasonal influenza vaccines in 20042008 was independently associated with protection in a recent study [19]. However, the population in that study comprised active-duty military service members, a high proportion of whom were younger than 40 years (94.3\%), and with vaccination rates over $80 \%$. Other study of institutionalized elderly patients in Hong Kong [20] found that dual vaccination of pandemic influenza A (H1N1) 2009 virus and seasonal influenza reduced allcause hospitalization, as well as hospitalization for fever on admission and for pneumonia, compared with seasonal vaccination alone. An additive effect of seasonal influenza and pneumococcal vaccination has also been described on all-cause mortality and mortality from pneumonia or from vascular causes [21]. However, in these two studies the majority of the population were women $(>60 \%)$, no laboratory-confirmed influenza infection was required, and the etiology of pneumonia (viral or bacterial) was not specified. Furthermore, the results cannot be extrapolated to all elderly persons not living in nursing homes.

The analysis of both the whole population and of patients with a vaccine indication revealed a longer hospital stay and greater use of antibiotics among immunized patients. These data do not support the idea that the influenza vaccine can lead to savings in terms of hospitalization costs. However, it should be noted that although there is widespread evidence of the cost-effectiveness of influenza vaccination $[33,34]$ this need not necessarily be the case in a particular high-risk population, such as ours, or in the context of an influenza pandemic.

Similar reasoning can be applied to explain the differences observed between patients vaccinated and those not vaccinated against pneumococcus. Once again, immunized patients tended to be older men with comorbidities, and they had more severe episodes. A high proportion of them also needed noninvasive ventilation. There were no differences with respect to the other outcome variables or mortality, although this may simply be due to the low number of immunized subjects. In our study only 28 patients had received both vaccines, even though there is some evidence that the benefits of influenza and pneumococcal vaccination may be additive in preventing hospitalization and death in high-risk groups, and even cost-effective [35-37].

It should be emphasized that bacterial infections tended to be less frequent among vaccinated patients in the present study. Only two pneumococcal infections were microbiologically documented among those who received influenza vaccine, and neither of them was invasive. Moreover, no pneumococcal infection was diagnosed among subjects who were immunized for $S$. pneumoniae. Although clinical studies have produced conflicting results regarding the utility of pneumococcal vaccination in some populations [38- 40] our data may support vaccination for high-risk patients.

Our study does have a number of limitations. Firstly, we only included hospitalized patients, who represent only a small proportion of those with influenza A(H1N1) pdm09 infection and who are likely to have a more severe clinical presentation and worse prognosis. A second limitation concerns the fact that we reviewed the effect of seasonal influenza vaccine on the clinical presentation and outcome of influenza $A(H 1 N 1)$ pdm09 infection in two different periods, the pandemic one and the first post-pandemic season, considering further that in the second period the seasonal influenza vaccine contained the H1N1 strain. However, in this second period we only had data for pneumonia episodes. It is also acknowledged that most of the vaccination data were self-reported, although there is evidence that self-reports, at least regarding influenza vaccination, are highly sensitive and evince a high level of agreement [17]. The strengths of this work include the fact that it was a multicenter study involving a large cohort of patients from 14 hospitals located in seven cities from across Spain. It is therefore reasonable to assume that our data offer a fair reflection of immunization practices, and their influence on clinical outcomes in different types of patients who were admitted with influenza A(H1N1) pdm09 infection to major centers during that period. However, it should be noted that for some outcome variables the small number of events meant that the analysis had insufficient statistical power to identify significant differences.

\section{Conclusion}

Vaccination rates against influenza and pneumococcus were low in hospitalized patients with influenza A(H1N1) pdm09 infection. In addition, no significant differences in outcomes between vaccinated and non-vaccinated patients were documented. However, vaccinated patients tended to have fewer $S$. pneumoniae bacterial co-infections.

\section{Funding}

This work was supported by Spain's Ministerio de Ciencia e Innovación, Instituto de Salud Carlos III, Programa de Investigación sobre gripe A/H1N1 [GR09/0014] and Ministerio de Economía y Competitividad, Instituto de 
Salud Carlos III - co-financed by European Development Regional Fund "A way to achieve Europe" ERDF, Spanish Network for the Research in Infectious Diseases (REIPI $\mathrm{RD} 12 / 0015)$. Dr. Viasus is the recipient of a research grant from REIPI.

\section{Conflict of interest}

The authors wish to express that they have no conflict of interest.

\section{List of author affiliations}

${ }^{1}$ Hospital Son Llàtzer, Palma de Mallorca, Spain

${ }^{2}$ Hospital Universitario Son Espases, Palma de Mallorca, Spain

${ }^{3}$ Hospital Universitari de Bellvitge - IDIBELL, University of Barcelona, Barcelona, Spain

${ }^{4}$ Hospital Universitario Virgen Macarena, Sevilla, Spain

${ }^{5}$ Hospital San Pedro - Centro de Investigación Biomédica de La Rioja (CIBIR), Logroño, Spain

${ }^{6}$ Hospital Universitario Reina Sofía - IMIBIC, University of Córdoba, Córdoba, Spain

${ }^{7}$ Hospital Universitario La Paz - IDIPAZ, Madrid, Spain

${ }^{8}$ Hospital Universitario Virgen del Rocio, Sevilla, Spain

${ }^{9}$ SCIAS - Hospital de Barcelona, Barcelona, Spain

${ }^{10}$ Hospital Universitario Marqués de Valdecilla, Santander, Spain

\section{References}

[1] Kuiken T, Taubenberger JK (2008) Pathology of human influenza revisited. Vaccine 26:D59-D66.

[2] Morens DM, Taubenberger JK, Fauci AS (2008) Predominant role of bacterial pneumonia as a cause of death in pandemic influenza: implications for pandemic influenza preparedness. J Infect Dis 198:962-70.

[3] Klugman KP, Astley CM, Lipsitch M (2009) Time from illness onset to death, 1918 influenza and pneumococcal pneumonia. Emerg Infect Dis 15:346-347.

[4] Viasus D, Paño-Pardo JR, Pachón J, Campins A, López-Medrano F, et al. (2011) Factors associated with severe disease in hospitalized adults with pandemic (H1N1) 2009 in Spain. Clin Microbiol Infect 17:738-746.

[5] Viasus D, Paño-Pardo JR, Pachón J, Riera M, López-Medrano F, et al. (2011) Pneumonia complicating pandemic (H1N1) 2009: risk factors, clinical features, and outcomes. Medicine (Baltimore) 90:328-336.

[6] Viasus D, Cordero E, Rodríguez-Baño J, Oteo JA, Fernández-Navarro A, et al. (2012) Changes in epidemiology, clinical features and severity of influenza A (H1N1) 2009 pneumonia in the first postpandemic influenza season. Clin Microbiol Infect 18:E55-62.

[7] McCullers JA (2006) Insights into the interaction between influenza virus and pneumococcus. Clin Microbiol Rev 19:571-582.

[8] Kash JC, Walters KA, Davis AS, Sandouk A, Schwartzman LM, et al. (2011) Lethal synergism of 2009 pandemic H1N1 influenza virus and Streptococcus pneumoniae coinfection is associated with loss of murine lung repair responses. MBio 2.pii:e00172-11.

[9] Advisory Committee on Immunization Practices (2012) Recommended adult immunization schedule: United States, 2012. Ann Intern Med 156:211-217.

[10] Mahmud S, Hammond G, Elliott L, Hilderman T, Kurbis C, et al. (2011) Effectiveness of the pandemic H1N1 influenza vaccines against laboratory-confirmed H1N1 infections: population-based case-control study. Vaccine 29:7975-7981.
[11] Horiya M, Hisano M, Iwasaki Y, Hanaoka M, Watanabe N, et al. (2011) Efficacy of double vaccination with the 2009 pandemic influenza A (H1N1) vaccine during pregnancy. Obstet Gynecol 118:887-894.

[12] Mascagni P, Vicenzi E, Kajaste-Rudnitski A, Pellicciotta G, Monti A, et al. (2012) Assessment of efficacy and safety of pandemic A/ H1N1/2009 influenza vaccine in a group of health care workers. Med Lav 103:220-229.

[13] Elkayam 0, Amir S, Mendelson E, Schwaber M, Grotto I, et al.(2011) Efficacy and safety of vaccination against pandemic 2009 influenza A (H1N1) virus among patients with rheumatic diseases. Arthritis Care Res (Hoboken) 63:1062-1067.

[14] Dikow R, Eckerle I, Ksoll-Rudek D, Hampel H, Schwenger V, et al. (2011) Immunogenicity and efficacy in hemodialysis patients of an AS03(A)-adjuvanted vaccine for 2009 pandemic influenza A(H1N1): a nonrandomized trial. Am J Kidney Dis 57:716-723.

[15] Blank PR, Schwenkglenks M, Szucs TD (2009) Vaccination coverage rates in eleven European countries during two consecutive influenza seasons. J Infect 58:446-458.

[16] Lu PJ, Nuorti JP (2010) Pneumococcal polysaccharide vaccination among adults aged 65 years and older, U.S., 1989-2008. Am J Prev Med 39:287-295.

[17] Johnson DR, Nichol KL, Lipczynski K (2008) Barriers to adult immunization. Am J Med 121:S28-35.

[18] Zimmerman RK, Nowalk MP, Terry MA, Raymund M, Tabbarah M, et al. (2008) Assessing disparities in adult vaccination using multimodal approaches in primary care offices: methodology. J Urban Health 85:217-227.

[19] Johns MC, Eick AA, Blazes DL, Lee SE, Perdue CL, et al. (2010) Seasonal influenza vaccine and protection against pandemic (H1N1) 2009-associated illness among US military personnel. PLoS One 5:e10722.

[20] Chan TC, Hung IF, Luk JK, Shea YF, Chan FH, et al. (2011) Efficacy of dual vaccination of pandemic H1N1 2009 influenza and seasonal influenza on institutionalized elderly: a one-year prospective cohort study. Vaccine 29:7773-7778.

[21] Chan TC, Hung IF, Luk JK, Shea YF, Chan FH, et al. (2012) Prevention of mortality and pneumonia among nursing home older adults by dual pneumococcal and seasonal influenza vaccination during a pandemic caused by novel pandemic influenza A (H1N1). J Am Med Dir Assoc 13:698-703.

[22] Fine MJ, Auble TE, Yealy DM, Hanusa BH, Weissfeld LA, et al. (1997) A prediction rule to identify low-risk patients with communityacquired pneumonia. N Engl J Med 336:243-250.

[23] Lim WS, van der Eerden MM, Laing R, Boersma WG, Karalus N, et al. (2003) Defining community acquired pneumonia severity on presentation to hospital: an international derivation and validation study. Thorax 58:377-382.

[24] Halm EA, Fine MJ, Marrie TJ, Coley CM, Kapoor WN, et al. (1998) Time to clinical stability in patients hospitalized with communityacquired pneumonia: implications for practice guidelines. JAMA 279:1452-1457.

[25] Varkey JB, Varkey AB, Varkey B (2009) Prophylactic vaccinations in chronic obstructive pulmonary disease: current status. Curr Opin Pulm Med 15:90-99.

[26] Jiménez-García R, Hernández-Barrera V, de Andres AL, JimenezTrujillo I, Esteban-Hernández J, et al. (2010) Gender influence in influenza vaccine uptake in Spain: time trends analysis (19952006). Vaccine 28:6169-6175.

[27] Jiménez-García R, Hernández-Barrera V, Carrasco-Garrido P, López de Andrés A, Pérez N, et al. (2008) Influenza vaccination coverages among children, adults, health care workers and immigrants in Spain: related factors and trends, 2003-2006. J Infect 57:472-480.

[28] Panda B, Stiller R, Panda A (2011) Influenza vaccination during pregnancy and factors for lacking compliance with current CDC guidelines. J Matern Fetal Neonatal Med 24:402-406. 
[29] Rodríguez-Rieiro C, Esteban-Vasallo MD, Domínguez-Berjón MF, Astray-Mochales J, Iniesta-Fornies D, et al. (2011) Coverage and predictors of vaccination against 2009 pandemic H1N1 influenza in Madrid, Spain. Vaccine 29:1332-1338.

[30] Nichol KL (2008) Efficacy and effectiveness of influenza vaccination. Vaccine 26:D17-22.

[31] Hak E, Buskens E, van Essen GA, de Bakker DH, Grobbee DE, et al. (2005) Clinical effectiveness of influenza vaccination in persons younger than 65 years with high-risk medical conditions: the PRISMA study. Arch Intern Med 165:274-280.

[32] Poole PJ, Chacko E, Wood-Baker RW, Cates CJ (2000) Influenza vaccine for patients with chronic obstructive pulmonary disease. Cochrane Database Syst Rev 25: CD002733.

[33] Nichol KL, Wuorenma J, von Sternberg T (1998) Benefits of influenza vaccination for low-, intermediate-, and high-risk senior citizens. Arch Intern Med 158: 1769-1776.

[34] Maciosek MV, Solberg LI, Coffield AB, Edwards NM, Goodman MJ (2006) Influenza vaccination health impact and cost effectiveness among adults aged 50 to 64 and 65 and older. Am J Prev Med 31:7279.

[35] Furumoto A, Ohkusa Y, Chen M, Kawakami K, Masaki H, et al. (2008) Additive effect of pneumococcal vaccine and influenza vaccine on acute exacerbation in patients with chronic lung disease. Vaccine 26:4284-4289.

[36] Smith KJ, Lee BY, Nowalk MP, Raymund M, Zimmerman RK (2010) Cost-effectiveness of dual influenza and pneumococcal vaccination in 50-year-olds. Vaccine 28:7620-7625.

[37] Gilchrist SA, Nanni A, Levine 0 (2012) Benefits and effectiveness of administering pneumococcal polysaccharide vaccine with seasonal influenza vaccine: an approach for policymakers. Am J Public Health 102:596-605

[38] Schenkein JG, Nahm MH, Dransfield MT (2008) Pneumococcal vaccination for patients with COPD: current practice and future directions. Chest 133:767-774.

[39] Pedersen RH, Lohse N, Østergaard L, Søgaard OS (2011) The effectiveness of pneumococcal polysaccharide vaccination in HIVinfected adults: a systematic review. HIV Med 12:323-333.

[40] Pitsiou GG, Kioumis IP (2011) Pneumococcal vaccination in adults: does it really work? Respir Med 105:1776-1783.

\section{Appendix}

Other members of the group are: Villoslada A, Pérez de la Blanca-Burgos M (Hospital Son Llàtzer); Peñaranda M (Hospital Universitario Son Espases); Niubó J (Hospital Universitari de Bellvitge); Rodríguez-Baño J, Fernández-Cuenca F, Toro MD (Hospital Universitario Virgen Macarena); García-Álvarez L (Hospital San Pedro - Centro de Investigación Biomédica de La Rioja); Torre-Cisneros J, Casal M, Causse M, GutiérrezAroca J, Lara R, Rivero A (Hospital Universitario Reina Sofía - IMIBIC, University of Córdoba); Romero-Gómez MP, Martín-Quirós A (Hospital Universitario La Paz - IDIPAZ); Pachón J, Aydillo T, Pérez-Romero P (Hospital Universitario Virgen del Rocio); Ortega L (SCIAS - Hospital de Barcelona); Casillas-Ruiz C, Fuente CG, M. Gutiérrez-Cuadra (Hospital Universitario Marqués de Valdecilla); Segura F (Hospital Parc Taulí); Francisco López-Medrano (Hospital Universitario 12 de Octubre); Moreno A (Hospital Clínic de Barcelona); Muñez-Rubio E (Hospital Universitario Puerta de Hierro). 